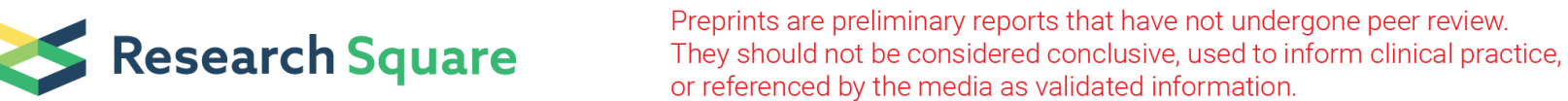

\section{Development and Testing of a Novel Universal Transdermal Drug Delivery Platform to Reduce Medication Nonadherence}

Gautam Ramesh ( $\square$ gramesh@ucsd.edu )

University of California San Diego School of Medicine https://orcid.org/0000-0003-4064-3353

\section{Ali Zamat}

UCSD Jacobs School of Engineering: University of California San Diego Jacobs School of Engineering

Srujan Vadlamudi

UC San Diego Division of Biological Sciences: University of California San Diego Division of Biological

Sciences

\section{Neiman Liu}

UC San Diego Division of Biological Sciences: University of California San Diego Division of Biological

Sciences

\section{Praveen Akuthota}

University of California San Diego Department of Medicine

\section{Short Report}

Keywords: Transdermal, Drug, Delivery, Nonadherence, Prednisolone, Polypharmacy

Posted Date: March 31st, 2021

DOl: https://doi.org/10.21203/rs.3.rs-375101/v1

License: (c) (i) This work is licensed under a Creative Commons Attribution 4.0 International License.

Read Full License 


\section{Abstract}

Medication nonadherence results in avoidable symptom exacerbations and hospitalization, causing thousands of deaths and billions of dollars in healthcare costs annually. Although pharmacological therapies are present for chronic diseases, lengthy and complicated medication regimens often have high rates of nonadherence. Transdermal drug delivery is a potential method of reducing medication nonadherence as it allows for long-course medication regimens to be simplified to a one-time epidermal patch. We have engineered a transdermal drug delivery system on a customizable platform to longitudinally deliver a wide variety of soluble small-molecule drugs. As a proof of concept, we have chosen to administer long-course corticosteroids for chronic inflammatory disease via epidermal patch. The transdermal drug delivery system has an integrated tapering mechanism to avoid end-of-course steroid dependence and can incorporate other small-molecule medications required for cases of polypharmacy. To date, there have been no successful attempts to develop long-term steroid patches for systemic delivery which can be attributed to issues with patch adhesion and skin irritation over extended periods of time. We have analyzed such challenges and have designed a proof of concept system to overcome these obstacles in order to provide patients with a simple, inexpensive, and effective solution to complicated and long-term treatment regimens.

\section{Introduction}

Medication nonadherence, or the patient's failure to follow the therapeutic regimen recommended by their healthcare provider, places an immense avoidable economic burden on the health system[1]. In the United States alone, up to $\$ 300$ billion of avoidable health care costs have been attributed to nonadherence, representing up to $50 \%$ of treatment failures and $10 \%$ of total healthcare costs nationwide[2]'[3]. Threefourths of Americans do not take their medication as directed[4], and 125,000 deaths per annum can be attributed to medication nonadherence[5]. Nonadherence to prescribed medication is directly associated with continued deterioration of health[6] and poor therapeutic outcomes[7][8] for countless chronic illnesses[9]. Factors contributing to medication nonadherence include forgetfulness, complacency[10], complicated dosing, cumbersome or long-term regimens[11], [12][13], prohibitively high costs[14], poor understanding of instructions[15], and difficulty of use, among others[16]. Previous studies have recognized simplification of treatment plans[17] and modifying the patient-provider dynamic to improve patient education and resultant compliance[18], but these strategies vary in efficacy by disease and patient health literacy, demonstrating the urgent need for treatments optimized to circumnavigate nonadherence.

Transdermal drug delivery systems (TDDS) have long been shown to have better adherence over traditional routes of medication administration (inhaled[19], oral[20]). TDDS saw great improvements in precision and reproducibility of systemic drug administration towards the end of the $20^{\text {th }}$ century[21]. Since the advent of scopolamine (hyoscine) as a topical means to manage seasickness[22], TDDS have been developed using many common drugs, including nitroglycerin[23], clonidine[24], and estradiol[25], 
among nearly 20 FDA approved drugs[26]. Transdermal patches have been developed for dozens of diseases[21], and therapies for many chronic conditions have taken advantage of transdermal delivery, resulting in positive outcomes for diseases such as Alzheimer's disease[27], HIV[28], attention-deficit hyperactivity disorder[29], and chronic pain[30].

Replacing a pill or inhaler with a transdermal patch has the potential to reduce medication nonadherence by reducing frequency of dosing[21], likelihood of improper use, as well as making it easier to remember, and increasing convenience[20]. Transdermal patches offer numerous benefits over conventional routes of administration: it is noninvasive, pain-free, and self-administered, and eliminates spikes and troughs of drug concentration in the blood, leading to more constant plasma drug concentration[31], thereby allowing for constant systemic effect. Transdermal patches directly introduce the drug into the blood stream, immediately making the drug available for systemic action, increasing bioavailability, bypassing the hepatic first-pass metabolic effect and gastrointestinal tract, and circumventing any contraindications related to gastrointestinal and respiratory routes of administration, such as intestinal complications present in inflammatory bowel diseases[32], [33]. Given the ample benefits, transdermal drug delivery is a leading contender for the development of platforms to reduce medication nonadherence, as it would simplify delivery of existing medications.

In the case of chronic respiratory syndromes, improper use of inhalers and complex dosing regiments may increase medication nonadherence. Month-long steroid regimens are often prescribed to prevent exacerbations following Chronic Obstructive Pulmonary Disease (COPD)-related hospitalizations. Up to $50 \%$ of COPD patients do not take nebulized treatments - many patients overuse, underuse, forget, or deliberately alter their regimen[34], preferring simpler, less frequent administrations of oral or injected medicine. This makes COPD patients an ideal target population to test a novel TDDS to tackle medication nonadherence. An adhesive epidermal patch that is waterproof, light, durable, and discreet would reduce aversion from self-medication, and would not require diligent patient activity to ensure regular medication administration. There are currently no specific mechanisms in place to reduce medication nonadherence in patients with long-term steroid prescriptions. Our current model aims to simplify prednisolone delivery by transporting the drug directly into the bloodstream via a noninvasive skin patch. Prednisolone is a viable candidate for transdermal delivery as it is of low molecular weight and moderately lipophilic[35], [36]. Prednisolone is the downstream metabolite of the prodrug prednisone. Conventional oral administration of prednisone results in hepatic metabolism into prednisolone, the biologically active form. Using concentrated prednisolone and transdermal administration directly into the bloodstream reduces the first-pass hepatic metabolism effect and reduces the necessity of high pre-metabolic doses, both of which would be necessary when using oral prednisone. Prednisolone will be stored and introduced with a cocktail of chemical excipients to ensure skin-safety and reduce systemic side-effects. As transdermal systems offer an affordable and easy-to-use means of reducing medication nonadherence, a TDDS built on a customizable platform to deliver a wide variety of medications could fulfill the needs of countless patients. Using a TDDS to simplify the process of self-medication and reducing patient effort could be an efficient way of reducing medication nonadherence without depending on the patient's health literacy. 


\section{Formulation}

We have developed a discreet, durable transdermal drug delivery patch with an integrated tapering mechanism that will allow patients to apply a single patch for the entire duration of their medication regimen. By reducing the amount of patient interaction with the medication, and by essentially allowing the patient to forget about the therapy, we eliminate the major causes of medication nonadherence, which include forgetfulness and conscious decision-making in medication administration.

\subsection{Drug-in-Adhesive Matrix}

The drug-in-adhesive matrix is composed of several components: the corticosteroid we are using is prednisolone (MilliporeSigma, St. Louis, Missouri, USA), a metabolite of prednisone, that is commonly used as anti-inflammatory or immunosuppressant medication for numerous systemic conditions. The adhesive is BIO-PSA MD7-4602 silicone adhesive (Dow Corning, Midland, Michigan, USA) as it provides a high tack, non-toxic base for our TDDS and is commonly used in the clinical setting[37], [38]. Ethyl acetate (MilliporeSigma, St. Louis, Missouri, USA) is used to dilute the adhesive, control viscosity, and make the mixture homogenous before it is evaporated off by degassing. A small amount $(<10 \%)$ of DMSO (MilliporeSigma, St. Louis, Missouri, USA) is used to dissolve prednisolone into the solution as well as to enhance skin permeability[32], [39]-[42]. Polyoxyethylene (20) oleyl ether (MilliporeSigma, St. Louis, Missouri, USA) is a penetration enhancer and plasticizer, which allows us to control the consistency of our solution. Isopropyl myristate (MilliporeSigma, St. Louis, Missouri, USA) is another penetration enhancer used in our TDDS to further push medication into and past the skin[32], [43], [44].

Using proprietary combinations of these ingredients in our matrix allows us to control dosages that are released over allotted periods of time. Further layering of different concentrations of each component allows for time-mediated delivery of various concentrations of the drug. As we can modulate both the concentrations within each layer and the layering order, we can deliver numerous sequential doses of medication all in a single patch. Higher concentrations of prednisolone can be delivered and subsequently tapered to lower dosages as the layers pass into the skin. We can also vary the width of each layer of drug-in-adhesive matrix, as well as concentration of medication, penetration enhancers, and other excipients, offering control over temporal administration of a particular dose. With two or more drug-in-adhesive matrices, as medication is delivered through the skin, layers of progressively lower drug concentrations will pass into the bloodstream over time. The formulation is easily customizable as each excipient and non-pharmacologic component interacts amicably with a wide variety of corticosteroids. 


\begin{tabular}{|llll|}
\hline Component & Function & Category & Supplier \\
\hline Prednisolone & Medication & $\begin{array}{l}\text { Drug-in-Adhesive } \\
\text { Matrix }\end{array}$ & MilliporeSigma \\
$\begin{array}{l}\text { BIO-PSA MD7-4602 Silicone } \\
\text { Adhesive }\end{array}$ & Adhesive & $\begin{array}{l}\text { Drug-in-Adhesive } \\
\text { Matrix }\end{array}$ & Dow Corning \\
\hline Ethyl acetate & Solvent & $\begin{array}{l}\text { Drug-in-Adhesive } \\
\text { Matrix }\end{array}$ & MilliporeSigma \\
\hline Dimethyl sulfoxide & Solvent & $\begin{array}{l}\text { Drug-in-Adhesive } \\
\text { Matrix }\end{array}$ & MilliporeSigma \\
\hline $\begin{array}{l}\text { Polyoxyethelene (20) oleyl } \\
\text { ether }\end{array}$ & Penetration enhancer & $\begin{array}{l}\text { Drug-in-Adhesive } \\
\text { Matrix }\end{array}$ & MilliporeSigma \\
\hline Isopropyl myristate & Penetration enhancer & $\begin{array}{l}\text { Drug-in-Adhesive } \\
\text { Matrix }\end{array}$ & MilliporeSigma \\
\hline Tegaderm & Securement & Dressing & 3M \\
\hline Scotchpak 9723 & Polyester film laminate & Backing Layer & 3M \\
\hline Scotchpak 9755 & $\begin{array}{l}\text { Fluoropolymer-coated } \\
\text { polyester film }\end{array}$ & Release Liner & 3M \\
\hline
\end{tabular}

Table 1. Transdermal drug delivery system components. Listed are the materials comprising the drug-inadhesive matrix, as well as the materials used as the dressing, backing layer, and release liner. Each component is the current industry standard or next-generation equivalent in transdermal drug delivery system manufacturing, from companies such as $3 \mathrm{M}$ and Dow Corning which supply adhesives and liners for current FDA-approved transdermal drug delivery systems.

\subsection{Dressing}

Tegaderm is a dressing widely used in clinical settings, often to secure and protect intravenous lines and catheter sites, as well as to cover wounds. We are using Tegaderm (3M, Maplewood, Minnesota, USA) to secure the patch to the site of application. Tegaderm conforms well to the skin that it is applied to, and increases skin hydration to promote transdermal penetration[45]. This ensures a watertight seal while protecting the TDDS from the environment. The Tegaderm material is flexible and comfortable, allowing the patient full range of mobility; at the same time, durability of the patch is not sacrificed.

\subsection{Backing Layer}

The backing layer we have chosen is the 3M Scotchpak 9723 Backing Polyester Film Laminate (3M, Maplewood, Minnesota, USA). The backing layer was specifically chosen because of its inherent properties and interactions with our drug-in-adhesive matrix, including good oxygen and moisture-vapor transmission[38]. This means that comfort for the patient is improved as the skin on which the TDDS is applied will not dry out as easily as with other backing layers. The backing layer also has high enhancer 
resistance, improving transdermal drug delivery. The backing layer is heat sealable, and formulated to resist leaching of the drug.

\subsection{Release Liner}

The release liner we have chosen is the 3M Scotchpak 9755 Fluoropolymer Coated Polyester Film (3M, Maplewood, Minnesota, USA). The fluoropolymer coating allows for complete separation of our drug-inadhesive matrix from the liner when the patch is peeled, assuring that no medication is lost in the process of preparing and applying the patch. The liner has also been found to maintain chemical stability and is compatible with our chosen silicone adhesive[37], [46].

\subsection{Tapering}

The drug delivery tapering mechanism is integrated into the TDDS by layering drug-in-adhesive matrices of varied drug concentration in a single TDDS, so that the patient does not have to remember to replace the patch with a lower dosage patch in order to achieve tapering. The components of the TDDS, including the high tack adhesive, that will prevent reduction of adhesion to skin over time, and a flexible and breathable backing layer that allows for long term comfort, address the FDA's main concerns about TDDS in today's market[47]

\section{Formulation And Testing}

Patients have shown through numerous studies over a broad range of different medical conditions that the adherence rate to TDDS-based delivery systems is up to $90.3 \%$, over $20 \%$ greater than other forms of drug delivery[19]. Clinical trials are necessary to confirm that our specific TDDS also reduces medication nonadherence in the target patient population.

The prednisolone, penetration enhancers, and other excipients were dissolved into the silicone adhesive. The current prototype is a drug-in-adhesive matrix, where all components are incorporated into monolithic strata between the release liner and the backing layer (Table 1). The 3M backing layer and release liner and the Dow Corning adhesive are the most effective components currently available on the market (highest tack, medical grade, most durable, compatible with excipients). Other TDDS currently use similar Dow Corning adhesives, with different solvents (heptane vs. ethyl acetate) and different bases (acrylic vs. silicone), and similar release liners (fluorosilicone vs. other fluoropolymer).

The patch solution is cured onto the backing layer, and a removable release liner was placed atop in order to maintain sterility and adhesion. The release liner was tested through repeated peeling to verify that the drug/adhesive solution maintains attached to the backing layer (data not shown). The surrounding Tegaderm dressing ensures that the patch will securely adhere to the skin of the patient for one week. The adhesive properties of the patch augment those of the Tegaderm assuring the entire system will be secured properly through physical activity. Moreover, the waterproof properties of the Tegaderm dressing further ensure strong adhesion to the skin for over seven days of daily activity (Fig. 1). 
The final TDDS prototype was placed on pig skin (Midwest Research Swine LLC, Glencoe, Minnesota, USA) for 1 week. The adhesive properties of the patch were maintained for at least seven days, as the patch was forcibly peeled off the skin for removal at this time-point (repeated twice more with similar results).

To test the medication administration ability of this system, in vitro testing was conducted by placing a 1 $\mathrm{cm}^{2}$ square patch on top of pig skin within a Franz Cell simulating physiological conditions at $37^{\circ} \mathrm{C}$ with a constant flow of phosphate buffered saline. Preliminary efficacy of the TDDS was determined over short periods of time. Samples were taken out of the cell at various time points and prednisolone concentrations were analyzed via Enzyme Linked Immunosorbent Assay (ELISA, MyBioSource, San Diego, CA, USA). The prednisolone ELISA kit is a competitive assay that comes precoated with a monoclonal anti-prednisolone antibody and utilizes a prednisolone-HRP conjugate as an inhibitor antigen. Samples are co-incubated with the conjugated prednisolone-HRP antigen, allowing for the prednisolone in the sample or the HRP conjugate to competitively bind to the well-bound antibody. After decanting and washing, an HRP substrate enzyme is added to the wells initiating a reaction that results in a blue colored complex. A stop solution is then added in order to halt the reaction, resulting in a yellow colored complex. Using a microplate reader, the wells are measured spectrophotometrically at $450 \mathrm{~nm}$. The intensity of the color is inversely proportional to the concentration of prednisolone, which is found using a standard curve plotted from the measurements of the provided concentration standards, allowing for the quantification of prednisolone concentration.

Prednisolone concentration increased over a tested seven day period in which our prototype TDDS delivered medication through pig skin and into the receptor chamber of the Franz Cell. Measurements were taken in triplicate and averaged once quantified at time points of hours $0,1,2,4$, and 8. Prednisolone was delivered at a constant rate over this period, indicated by a positive linear trend of prednisolone concentration within the receptor chamber.

To further explore the efficacy and long term ability of the TDDS, samples were taken over week-long period to be further analyzed through mass spectrometry (MS). MS accurately detects and quantifies molecules and chemical compounds through the measurement of mass-to-charge ratio of ions. The liquid samples from the Franz Cell receptor chamber were charged before being accelerated within the mass spectrometer and subsequently deflected by a magnetic field. The molecules of the sample can be correlated using known standards. Samples were taken from the Franz Cell at hours 2, 4, 8, 36, 72 (day 3), 144 (day 6), and 192 (day 8) and analyzed using MS courtesy of a partner laboratory. Concentrations of prednisolone were plotted against their time points and a model of the transdermal activity of the TDDS was created using semilogarithmic, nonlinear regression (Fig. 2). Over a week-long period, prednisolone concentration within the Franz Cell began to flatten out after a brief period of relatively quick transdermal activity.

\section{Discussion}


Our TDDS is a unique extension of the already existing generic transdermal medication delivery system. Previously patented TDDS currently in the market do not offer tapering and flexibility with the use of different dosages and medications, novel inclusions in our TDDS prototype. There exist no specific nonadherence-reducing systems in place for long-term steroid regimens. Currently, the standard method of delivering prednisone is through oral tablets, which patients often forget to take - our TDDS is a replacement for this method of administration. The first-pass hepatic metabolism effect also requires that oral pills contain more medication than our TDDS, as drug concentration is greatly reduced when it is passes through the digestive tract and liver. There are presently fewer than 30 transdermal drug delivery systems on the market, most commonly for birth control and to alleviate nicotine addiction - our TDDS is implementing a therapeutic for an extremely common corticosteroid with a complex tapering regimen, and will eventually encompass other long-term medications with high rates of nonadherence, a market void that has not been filled, even with documented evidence of higher adherence to TDDS than traditional therapies.

Non-esterized prednisolone has been shown to have negligible binding within the skin in mouse models[48]. Although transdermal delivery of steroids such as estradiol and testosterone have been well studied, local effects of prednisolone will need to be evaluated in animal models and clinical testing of our TDDS as few studies have evaluated any dermal side-effects of prednisolone. Matrix patches are typically associated with fewer skin reactions than earlier reservoir patches due to improved air circulation and absence of alcohol-based solvents[49]. Studies on similarly designed TDDS delivering hormones have established low risk of skin irritation, pruritis, urticaria, and did not notice local effects of the medication[50]-[52]. Mild sub-acute irritation and erythema have been observed in patch formulations lacking drug as well, indicating that occlusion by the patch itself may contribute to skin irritation, while any local dermal effects by the drug were reversible[53]. As prednisolone can be used as a topical anti-inflammatory (although hydrocortisone is preferred), local adverse dermal effects are rare, and reported in less than $4 \%$ of cases[54]. Data from the National Poison Data System evaluating TDDS safety suggests that Fentanyl-containing patches are more likely to be abused and are more highly associated with death, a drug that is not present in the described TDDS[55].

Pharmacokinetic parameters obtained from transdermal prednisolone administration in rats have shown that a $3 \mathrm{mg} / 10 \mathrm{~cm}^{2} /$ patch, testing $30 \mathrm{~cm}^{2}$ patches (about $9 \mathrm{mg}$ prednisolone per patch), delivers the same peak plasma concentration as a $10 \mathrm{mg} / \mathrm{kg}$ oral dose of prednisolone[56]. The oral formulation peaks in plasma concentration at $1000 \mathrm{ng} / \mathrm{mL}$ within the first hour and diminishes to $<50 \mathrm{ng} / \mathrm{mL}$ within 5 hours. The patch maintains a plasma concentration between $100-1000 \mathrm{ng} / \mathrm{mL}$ over the course of 24 hours, with a peak after 7 hours and a gradual decline afterwards.

Current platforms for TDDS include iontophoretic systems, which use an electrical current to drive transdermal drug delivery; these are currently in development and require either a triboelectric nanogenerator or power supply to modulate drug delivery[57]. Microneedle technologies have successfully delivered vaccines but are more complicated to manufacture and require microscopic penetration of the stratum corneum[58]. Proniosomal patches offer benefits over liposome-based TDDS, 
but are currently rudimentary and have not been shown to be effective with a wide variety of drugs[59], [60]. Current technology in transdermal steroid delivery, outside of matrix-based patches, is currently comprised of liposome-mediated delivery and nanoparticle formulations[61], [62]. Drug-in-adhesive matrices are easier to manufacture and are backed by decades of research and development; furthermore, their individual components are more widely available and less expensive due to commercial competition and non-proprietary availability.

We expect that our TDDS will be reimbursable by Medicare and Medicaid. Currently oral prednisone pills are either fully or partially covered by Medicare Part D. Because prednisone is a widely used drug that treats numerous different conditions, prednisone is often covered by Medicare. Medicare Part D plans specify coverage over drugs that fall into six categories: antidepressants, antipsychotics, anticonvulsants, antiretrovirals (AIDS treatment), immunosuppressants and anticancer. Prednisone and its metabolite prednisolone are both anti-inflammatory drugs and therefore fall under the category of immunosuppressants. TDDS which are no longer covered by Medicare/Medicaid are transdermal drug delivery systems that are not primarily aimed to alleviate debilitating conditions such as systemic inflammation, as our TDDS will.

Validation of our prototype and clinical usage of our platform will require: further in-vitro testing of liquid chromatography-mass spectrometry of the release liner to ensure absence of steroid, digital adhesion testing in order to quantify the adhesive properties of the TDDS at a variety of time points, massmanufacturing technique to produce sufficient units to begin in vivo testing, and animal testing and subsequent clinical trials

\section{Declarations}

Funding: G.R., A.Z., S.V., and N.L recognize initial funding from the Frontiers of Innovation Scholars Program at the University of California, San Diego. P.A. reports no funding for this manuscript.

Conflicts of interest/Competing interests: P.A. has received research support from the National Institutes of Health, has received research support and consultancy fees from and has been on advisory boards for AstraZeneca and GlaxoSmothKline; has received consultancy fees from Ambrx; receives royalties from UpToDate; and has received honoraria from WebMD/Medscape, AHK, Prime CME, Rockpointe, MJH Life Sciences, and Vindico. G.R., A.Z., S.V., and N.L. report no conflicts of interest or competing interests.

Availability of data and material: All data and material are available on request.

Code availability: Not applicable

Authors' contributions: G.R., A.Z., S.V., and P.A. contributed to the conception, design, and experimental execution and manuscript writing and editing. N.L contributed to the experimental execution of this manuscript.

Ethics approval: Not applicable 
Consent to participate: Not applicable

Consent for publication: Not applicable

Acknowledgements: The authors would like to thank Dr. Laura Crotty-Alexander for her help in initiating this project and Dr. Louis-Félix Nothias for his help with mass spectrometry.

\section{References}

[1] L. R. Martin, S. L. Williams, K. B. Haskard, and M. R. Dimatteo, "The challenge of patient adherence," Ther Clin Risk Manag, vol. 1, no. 3, pp. 189-199, Sep. 2005.

[2] A. O. luga and M. J. McGuire, "Adherence and health care costs," Risk Manag Healthc Policy, vol. 7, pp. 35-44, Feb. 2014, doi: 10.2147/RMHP.S19801.

[3] R. M. Benjamin, "Medication Adherence: Helping Patients Take Their Medicines As Directed," Public Health Rep, vol. 127, no. 1, pp. 2-3, 2012.

[4] New England Healthcare Institute, "Thinking outside the pillbox: a system-wide approach to improving patient medication adherence for chronic disease.," NEHI Research Brief, Aug. 2009, Accessed: Aug. 19, 2020. [Online]. Available:

https://www.nehi.net/writable/publication_files/file/pa_issue_brief_final.pdf.

[5] R. McCarthy, "The price you pay for the drug not taken," Bus Health, vol. 16, no. 10, pp. 27-28, 30, 32-33, Oct. 1998.

[6] N. M. Siafakas and D. Bouros, "Consequences of poor compliance in chronic respiratory diseases," European Respiratory Journal, vol. 5, no. 1, pp. 134-136, Jan. 1992.

[7] W. J. Burman, D. L. Cohn, C. A. Rietmeijer, F. N. Judson, R. R. Reves, and J. A. Sbarbaro, "Noncompliance With Directly Observed Therapy for Tuberculosis: Epidemiology and Effect on the Outcome of Treatment," Chest, vol. 111, no. 5, pp. 1168-1173, May 1997, doi: 10.1378/chest.111.5.1168.

[8] S. Kane, D. Huo, J. Aikens, and S. Hanauer, "Medication nonadherence and the outcomes of patients with quiescent ulcerative colitis," Am. J. Med., vol. 114, no. 1, pp. 39-43, Jan. 2003, doi: 10.1016/s0002-9343(02)01383-9.

[9] K. S. Ingersoll and J. Cohen, "The impact of medication regimen factors on adherence to chronic treatment: a review of literature," J Behav Med, vol. 31, no. 3, pp. 213-224, Jun. 2008, doi: $10.1007 /$ s10865-007-9147-y.

[10] A. G. Bulloch, C. E. Adair, and S. B. Patten, "Forgetfulness: A Role in Noncompliance with Antidepressant Treatment," Can J Psychiatry, vol. 51, no. 11, pp. 719-722, Oct. 2006, doi: 10.1177/070674370605101110. 
[11] G. S. Gaude, J. Hattiholi, and A. Chaudhury, "Role of health education and self-action plan in improving the drug compliance in bronchial asthma," J Family Med Prim Care, vol. 3, no. 1, pp. 33-38, Jan. 2014, doi: 10.4103/2249-4863.130269.

[12] J. R. Ickovics and A. W. Meisler, "Adherence in AIDS clinical trials: a framework for clinical research and clinical care," J Clin Epidemiol, vol. 50, no. 4, pp. 385-391, Apr. 1997, doi: 10.1016/s08954356(97)00041-3.

[13] C. Penn, J. Watermeyer, and M. Evans, "Why don't patients take their drugs? The role of communication, context and culture in patient adherence and the work of the pharmacist in HIV/AIDS," Patient Educ Couns, vol. 83, no. 3, pp. 310-318, Jun. 2011, doi: 10.1016/j.pec.2011.02.018.

[14] W. H. Shrank et al., "The implications of choice: prescribing generic or preferred pharmaceuticals improves medication adherence for chronic conditions," Arch. Intern. Med., vol. 166, no. 3, pp. 332-337, Feb. 2006, doi: 10.1001/archinte.166.3.332.

[15] K. F. Foreman et al., "Impact of a text messaging pilot program on patient medication adherence," Clin Ther, vol. 34, no. 5, pp. 1084-1091, May 2012, doi: 10.1016/j.clinthera.2012.04.007.

[16] M. R. DiMatteo, "Variations in patients' adherence to medical recommendations: a quantitative review of 50 years of research," Med Care, vol. 42, no. 3, pp. 200-209, Mar. 2004, doi: 10.1097/01.mlr.0000114908.90348.f9.

[17] A. J. Claxton, J. Cramer, and C. Pierce, "A systematic review of the associations between dose regimens and medication compliance," Clin Ther, vol. 23, no. 8, pp. 1296-1310, Aug. 2001, doi: 10.1016/s0149-2918(01)80109-0.

[18] M. R. DiMatteo, "Evidence-based strategies to foster adherence and improve patient outcomes," JAAPA, vol. 17, no. 11, pp. 18-21, Nov. 2004.

[19] H. Mochizuki, Y. Nanjo, and H. Takahashi, "Better adherence to a transdermal tulobuterol patch than inhaled salmeterol in elderly chronic obstructive pulmonary disease patients," Geriatr Gerontol Int, vol. 13, no. 2, pp. 398-404, Apr. 2013, doi: 10.1111/j.1447-0594.2012.00916.x.

[20] P. G. Conaghan, M. Serpell, P. McSkimming, R. Junor, and S. Dickerson, "Satisfaction, Adherence and Health-Related Quality of Life with Transdermal Buprenorphine Compared with Oral Opioid Medications in the Usual Care of Osteoarthritis Pain," Patient, vol. 9, pp. 359-371, 2016, doi: 10.1007/s40271-016-0181-0.

[21] M. N. Pastore, Y. N. Kalia, M. Horstmann, and M. S. Roberts, "Transdermal patches: history, development and pharmacology," Br J Pharmacol, vol. 172, no. 9, pp. 2179-2209, May 2015, doi: 10.1111/bph.13059. 
[22] “Holling HE, McArdle B, Trotter WR (1944)," The James Lind Library, Jul. 12, 2013. https://www.jameslindlibrary.org/holling-he-mcardle-b-trotter-wr-1944/ (accessed Aug. 19, 2020).

[23] S. Sved, W. M. McLean, and I. J. McGilveray, "Influence of the method of application on pharmacokinetics of nitroglycerin from ointment in humans," J Pharm Sci, vol. 70, no. 12, pp. 1368-1369, Dec. 1981, doi: 10.1002/jps.2600701220.

[24] D. A. Sica and R. Grubbs, "Transdermal clonidine: therapeutic considerations," J Clin Hypertens (Greenwich), vol. 7, no. 9, pp. 558-562, Sep. 2005, doi: 10.1111/j.1524-6175.2005.04133.x.

[25] J. W. Goldzieher and R. E. Baker, "The percutaneous absorption of estradiol-17beta and progesterone," J. Invest. Dermatol., vol. 35, pp. 215-218, Oct. 1960.

[26] C. for D. E. and Research, "Approved Drug Products with Therapeutic Equivalence Evaluations (Orange Book)," FDA, Aug. 2020, Accessed: Aug. 19, 2020. [Online]. Available:

https://www.fda.gov/drugs/drug-approvals-and-databases/approved-drug-products-therapeuticequivalence-evaluations-orange-book.

[27] T. T. Nguyen, V. V. Giau, and T. K. Vo, "Current advances in transdermal delivery of drugs for Alzheimer's disease," Indian J Pharmacol, vol. 49, no. 2, pp. 145-154, 2017, doi: 10.4103/02537613.208143.

[28] A. S. Ham and R. W. Buckheit, "Current and emerging formulation strategies for the effective transdermal delivery of HIV inhibitors," Ther Deliv, vol. 6, no. 2, pp. 217-229, Feb. 2015, doi: 10.4155/tde.14.110.

[29] R. L. Findling and S. Dinh, "Transdermal Therapy for Attention-Deficit Hyperactivity Disorder with the Methylphenidate Patch (MTS)," CNS Drugs, vol. 28, no. 3, pp. 217-228, 2014, doi: 10.1007/s40263014-0141-y.

[30] M. Serpell, S. Tripathi, S. Scherzinger, S. Rojas-Farreras, A. Oksche, and M. Wilson, "Assessment of Transdermal Buprenorphine Patches for the Treatment of Chronic Pain in a UK Observational Study," Patient, vol. 9, pp. 35-46, 2016, doi: 10.1007/s40271-015-0151-y.

[31] N. Al-Tawil, I. Odar-Cederlöf, A.-C. Berggren, H. E. Johnson, and J. Persson, "Pharmacokinetics of transdermal buprenorphine patch in the elderly," Eur J Clin Pharmacol, vol. 69, no. 2, pp. 143-149, Feb. 2013, doi: 10.1007/s00228-012-1320-8.

[32] K. S. Paudel, M. Milewski, C. L. Swadley, N. K. Brogden, P. Ghosh, and A. L. Stinchcomb, "Challenges and opportunities in dermal/transdermal delivery," Ther Deliv, vol. 1, no. 1, pp. 109-131, Jul. 2010, doi: $10.4155 /$ tde.10.16.

[33] J. J. Berti and J. J. Lipsky, "Transcutaneous drug delivery: a practical review," Mayo Clin. Proc., vol. 70, no. 6, pp. 581-586, Jun. 1995, doi: 10.4065/70.6.581. 
[34] F. Blasi, F. Raddi, and M. Miravitlles, "Interactive Monitoring Service and COPD: Is it Possible to Reduce Nonadherence?," COPD, vol. 12, no. 3, pp. 227-232, Jun. 2015, doi:

$10.3109 / 15412555.2014 .933796$.

[35] J. D. Bos and M. M. Meinardi, "The 500 Dalton rule for the skin penetration of chemical compounds and drugs," Exp. Dermatol., vol. 9, no. 3, pp. 165-169, Jun. 2000, doi: 10.1034/j.16000625.2000.009003165.x.

[36] A. P. Funke, C. Günther, R. H. Müller, and R. Lipp, “In-vitro release and transdermal fluxes of a highly lipophilic drug and of enhancers from matrix TDS," J Control Release, vol. 82, no. 1, pp. 63-70, Jul. 2002, doi: 10.1016/s0168-3659(02)00105-0.

[37] M. H. Qvist, U. Hoeck, B. Kreilgaard, F. Madsen, and S. Frokjaer, "Release of chemical permeation enhancers from drug-in-adhesive transdermal patches," Int J Pharm, vol. 231, no. 2, pp. 253-263, Jan. 2002, doi: 10.1016/s0378-5173(01)00893-6.

[38] S. Banerjee, P. Chattopadhyay, A. Ghosh, P. Datta, and V. Veer, "Aspect of adhesives in transdermal drug delivery systems," International Journal of Adhesion and Adhesives, vol. 50, pp. 70-84, Apr. 2014, doi: 10.1016/j.jjadhadh.2014.01.001.

[39] R. J. Scheuplein and I. H. Blank, “Permeability of the skin,” vol. 51, p. 46, 1971.

[40] B. Bednarczyk-Cwynar, D. Partyka, and L. Zaprutko, "Simple amides of oleanolic acid as effective penetration enhancers," PLOS ONE, vol. 10, no. 5, p. e0122857, 2015, doi: 10.1371/journal.pone.0122857.

[41] A. C. Williams and B. W. Barry, "The enhancement index concept applied to terpene penetration enhancers for human skin and model lipophilic (oestradiol) and hydrophilic (5-fluorouracil) drugs," International Journal of Pharmaceutics, vol. 74, no. 2, pp. 157-168, Aug. 1991, doi: 10.1016/03785173(91)90232-D.

[42] R. B. Walker and E. W. Smith, "The role of percutaneous penetration enhancers," Advanced Drug Delivery Reviews, vol. 18, no. 3, pp. 295-301, Feb. 1996, doi: 10.1016/0169-409X(95)00078-L.

[43] A. Eichner et al., "Influence of the penetration enhancer isopropyl myristate on stratum corneum lipid model membranes revealed by neutron diffraction and 2H NMR experiments," Biochim Biophys Acta Biomembr, vol. 1859, no. 5, pp. 745-755, May 2017, doi: 10.1016/j.bbamem.2017.01.029.

[44] T. N. Engelbrecht, B. Demé, B. Dobner, and R. H. H. Neubert, "Study of the influence of the penetration enhancer isopropyl myristate on the nanostructure of stratum corneum lipid model membranes using neutron diffraction and deuterium labelling," Skin Pharmacol Physiol, vol. 25, no. 4, pp. 200-207, 2012, doi: 10.1159/000338538.

[45] H. A. E. Benson, "Skin Structure, Function, and Permeation," in Topical and Transdermal Drug Delivery, John Wiley \& Sons, Ltd, 2012, pp. 1-22. 
[46] D. G. Maillard-Salin, P. Bécourt, and G. Couarraze, "Physical evaluation of a new patch made of a progestomimetic in a silicone matrix," Int J Pharm, vol. 199, no. 1, pp. 29-38, Apr. 2000, doi:

10.1016/s0378-5173(00)00357-4.

[47] N. Sadrieh, "Challenges in the Development of Transdermal Drug Delivery Systems," FDA Advisory Committee for Pharmaceutical Science and Clinical Pharmacology, Aug. 2009.

[48] T. Hikima and K. Tojo, "Binding of prednisolone and its ester prodrugs in the skin," Pharm Res, vol. 14, no. 2, pp. 197-202, Feb. 1997, doi: 10.1023/a:1012052827872.

[49] J.-Y. Reginster, Y. Donazzolo, N. Brion, and R. Lins, “Estradiol pharmacokinetics after transdermal application of patches to postmenopausal women: matrix versus reservoir patches," null, vol. 3, no. 3, pp. 168-175, Jan. 2000, doi: 10.1080/13697130008500093.

[50] A. M. Kaunitz, D. Portman, C. L. Westhoff, D. R. Mishell, D. F. Archer, and M. Foegh, "New contraceptive patch wearability assessed by investigators and participants in a randomized phase 3 study," Contraception, vol. 91, no. 3, pp. 211-216, Mar. 2015, doi: 10.1016/j.contraception.2014.11.013.

[51] D. R. Friend, "Transdermal delivery of levonorgestrel," Medicinal Research Reviews, vol. 11, no. 1, pp. 49-80, 1991, doi: https://doi.org/10.1002/med.2610110105.

[52] S. Logsdon, J. Richards, and H. A. Omar, "Long-Term Evaluation of the Use of the Transdermal Contraceptive Patch in Adolescents," ScientificWorldJournal, vol. 4, pp. 512-516, Jul. 2004, doi: 10.1100/tsw.2004.107.

[53] D. R. Friend, S. J. Phillips, and J. R. Hill, "Cutaneous effects of transdermal levonorgestrel," Food and Chemical Toxicology, vol. 29, no. 9, pp. 639-646, Jan. 1991, doi: 10.1016/0278-6915(91)90147-Y.

[54] L. Frank, "Prednisolone Topically and Systemically: A Clinical Evaluation in Selected Dermatoses: Preliminary Report," AMA Arch Derm, vol. 72, no. 6, p. 547, Dec. 1955, doi:

10.1001/archderm.1955.03730360053005.

[55] S. L. Thornton and M. A. Darracq, "Patch Problems? Characteristics of Transdermal Drug Delivery System Exposures Reported to the National Poison Data System," J. Med. Toxicol., vol. 16, no. 1, pp. 3340, Jan. 2020, doi: 10.1007/s13181-019-00723-0.

[56] A. Isowaki, A. Ohtori, Y. Matsuo, and K. Tojo, "Drug delivery to the eye with a transdermal therapeutic system," Biol Pharm Bull, vol. 26, no. 1, pp. 69-72, Jan. 2003, doi: 10.1248/bpb.26.69.

[57] C. Wu et al., "Self-Powered Iontophoretic Transdermal Drug Delivery System Driven and Regulated by Biomechanical Motions," Advanced Functional Materials, vol. 30, no. 3, p. 1907378, 2020, doi: https://doi.org/10.1002/adfm.201907378. 
[58] M. Azmana, S. Mahmood, A. R. Hilles, U. K. Mandal, K. A. Saeed Al-Japairai, and S. Raman, "Transdermal drug delivery system through polymeric microneedle: A recent update," Journal of Drug Delivery Science and Technology, vol. 60, p. 101877, Dec. 2020, doi: 10.1016/j.jddst.2020.101877.

[59] M. H. Aboumanei and Ashgan. F. Mahmoud, "Design and development of a proniosomal transdermal drug delivery system of caffeine for management of migraine: In vitro characterization, 1311radiolabeling and in vivo biodistribution studies," Process Biochemistry, vol. 97, pp. 201-212, Oct. 2020, doi: 10.1016/j.procbio.2020.07.018.

[60] G. V. Radha, T. S. Rani, and B. Sarvani, "A review on proniosomal drug delivery system for targeted drug action," Journal of Basic and Clinical Pharmacy, vol. 4, no. 2, May 2015, Accessed: Nov. 10 , 2020. [Online]. Available: https://www.jbclinpharm.org/abstract/a-review-on-proniosomal-drug-deliverysystem-for-targeted-drug-action-1390.html.

[61] E. B. Souto et al., "SLN and NLC for topical, dermal, and transdermal drug delivery," Expert Opinion on Drug Delivery, vol. 17, no. 3, pp. 357-377, Mar. 2020, doi: 10.1080/17425247.2020.1727883.

[62] S. N. Shrotriya, N. S. Ranpise, and B. V. Vidhate, "Skin targeting of resveratrol utilizing solid lipid nanoparticle-engrossed gel for chemically induced irritant contact dermatitis," Drug Deliv. and Transl. Res., vol. 7, no. 1, pp. 37-52, Feb. 2017, doi: 10.1007/s13346-016-0350-7.

\section{Figures}




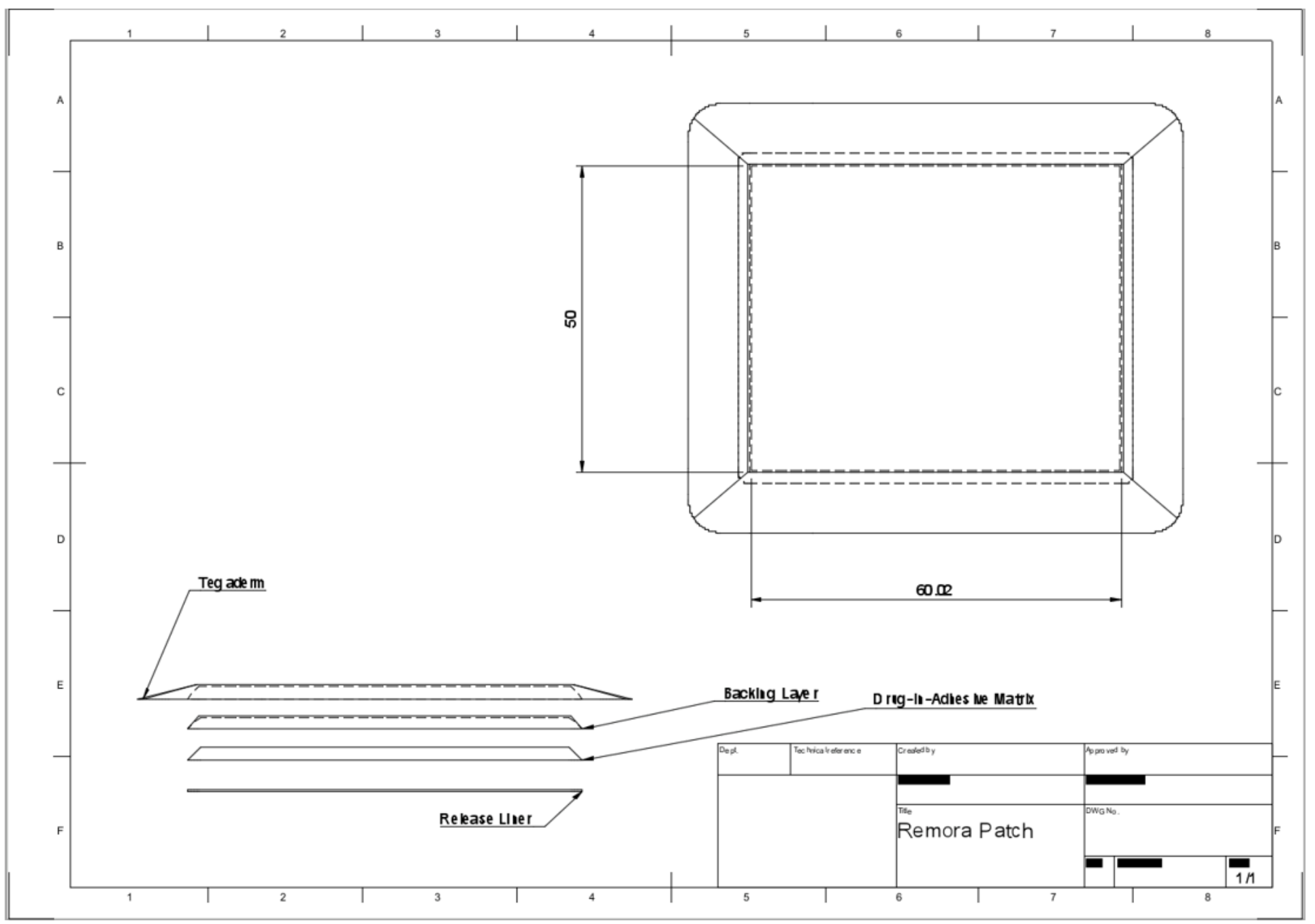

\section{Figure 1}

Transdermal drug delivery system blueprint. This schematic shows the mock-up of the patch components. Most superficially is the waterproof Tegaderm, providing external protection. Directly below is the backing layer, a specialized film onto which the drug-in-adhesive matrix is cured. The drug-inadhesive matrix houses the medication, penetration enhancers, and solvents. The final layer is the release liner, which is removed before application to the skin, exposing the adhesive matrix. The Tegaderm dimensions span one-half centimeter past the dimensions of the patch, securing directly to the skin. 


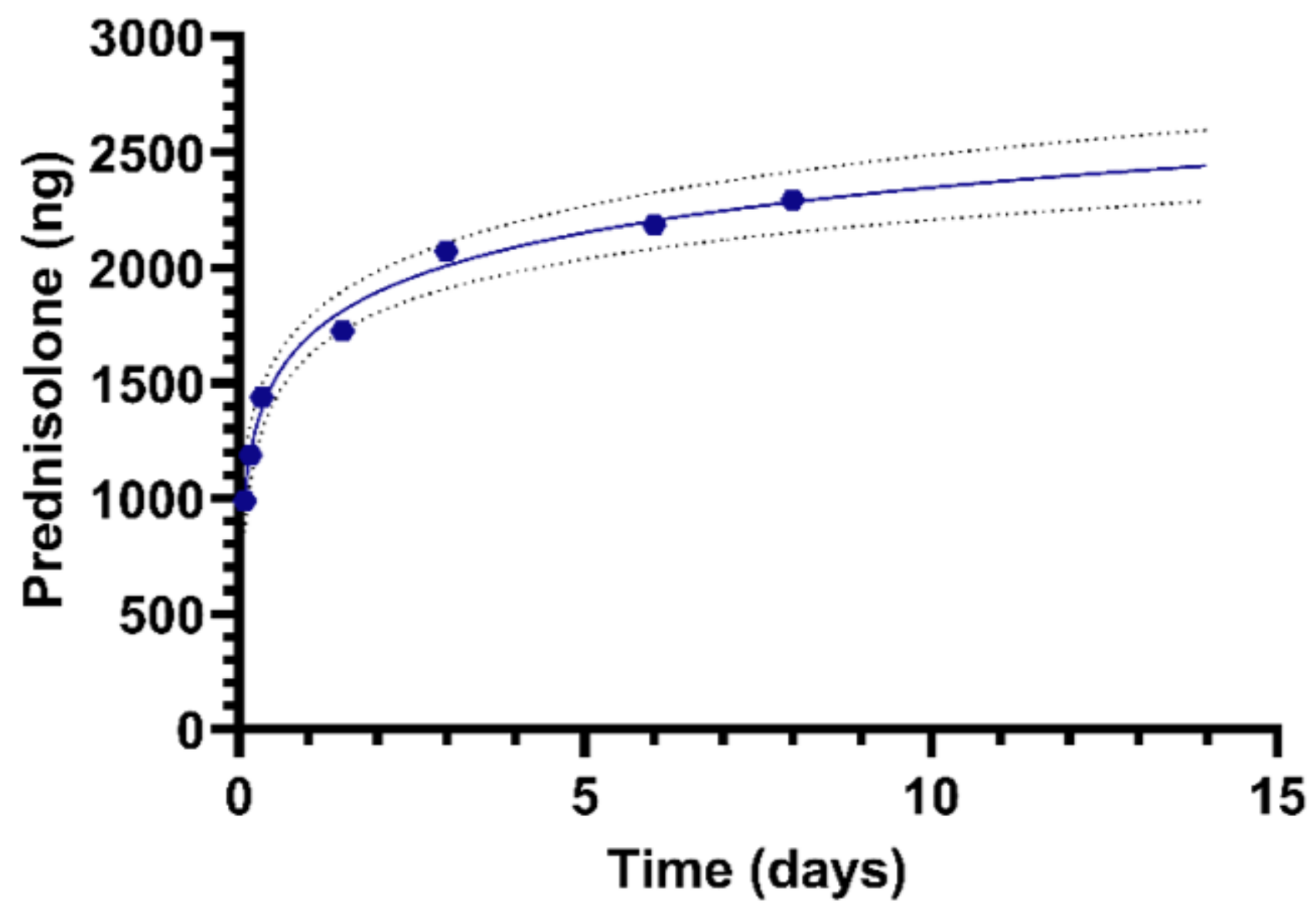

Figure 2

Prednisolone Concentration over time in Franz Cell Receptor Chamber. Samples were taken from the Franz Cell at Hours 2, 4, 8, 36, 72 (day 3), 144 (day 6), and 192 (day 8) and were analyzed and quantified via mass spectrometry. The plotted points signify actual concentration of prednisolone within the sample at the respective time point. The solid line is the graphed, semilogarithmic, nonlinear regression model of TDDS transdermal activity with equation $Y=1699+(648.5) \star \log (X)$, with the dotted lines indicating confidence bands in-between which the likely location of the true curve can be found at $99 \%$ confidence level.

\section{Supplementary Files}

This is a list of supplementary files associated with this preprint. Click to download.

- graphicalabstract.png 